\title{
On the static Casimir effect with parity-breaking mirrors
}

\author{
C. D. Fosco ${ }^{1, \mathrm{a}}$, M. L. Remaggi ${ }^{2}$ \\ ${ }^{1}$ Centro Atómico Bariloche and Instituto Balseiro, Comisión Nacional de Energía Atómica, 8400 Bariloche, Argentina \\ ${ }^{2}$ Facultad de Ciencias Exactas y Naturales, Universidad Nacional de Cuyo, 5500 Mendoza, Argentina
}

Received: 8 September 2016 / Accepted: 1 March 2017 / Published online: 10 March 2017

(c) The Author(s) 2017. This article is published with open access at Springerlink.com

\begin{abstract}
We study the Casimir interaction energy due to the vacuum fluctuations of the electromagnetic (EM) field in the presence of two mirrors, described by $2+1$ dimensional, generally nonlocal actions, which may contain both parity-conserving and parity-breaking terms. We compare the results with the ones corresponding to ChernSimons boundary conditions and evaluate the interaction energy for several particular situations.
\end{abstract}

\section{Introduction}

The Casimir effect [1-3] is usually regarded as one of the most remarkable macroscopic manifestations of the fluctuations (be they quantum or thermal) of a field when it is subjected to the non-trivial influence of external agents. The latter usually manifest themselves as boundary conditions, or as 'boundary terms' in the action for the field. These terms are, by definition, contributions depending only on the field and its derivatives on the boundary; therefore, they can be interpreted as due to singular terms (involving generalized functions) in the Lagrangian. The effect results from the interplay between those external agents ('mirrors') and the field fluctuations. In the static version of the effect, the one we are concerned with here, one considers time-independent boundary conditions or, equivalently, boundary terms which do not depend explicitly on time.

A variety of situations can be explored where this effect becomes relevant; a natural way to exhaust them all, is by either considering fluctuating fields of different nature for each boundary condition, or by studying the consequences of imposing different boundary conditions on each given field. In principle, both the mirrors' geometry and their intrinsic properties are relevant to the effect. Having in mind the latter, our aim here is to consider boundary actions containing both parity-conserving and parity-breaking terms, for an

\footnotetext{
a e-mail: fosco@cab.cnea.gov.ar
}

Abelian gauge field in $3+1$ dimensions, ${ }^{1}$ in the presence of two zero-width mirrors. A concrete realization of that kind of boundary term are the effective actions in $2+1$ dimensions which represent the quantum effects due to a Dirac field confined to the mirrors' world-volumes, and minimally coupled to the projection of the gauge field to the world-volume swept by the boundary. This kind of field theory, in particular its parity-breaking effects, arises naturally in the context of the quantum Hall effect [4]. As far as we know, experiments allowing for the realization of that effect in two parallel plates, and the simultaneous detection of the Casimir force between them, have not been yet implemented. The most difficult obstacle to do this is perhaps the need to have strong magnetic fields (from the quantum Hall effect side), without perturbing the delicate detection of the Casimir force.

Note that the Casimir effect due to Chern-Simons (C$\mathrm{S})$ boundary conditions has been studied since the pioneering work of reference [5], where it has been shown that the Casimir force may, for some choices of the parameters, become repulsive. It is our aim here to study the problem of including parity-breaking terms in the boundary action, as opposed to boundary conditions. The two approaches, although related, are essentially different, a fact that has been highlighted already in [5].

There have been several interesting developments related to this kind of system: the Casimir effect for a spherical region, with $\mathrm{C}-\mathrm{S}$ like boundary conditions due to the presence of a $\theta$ term, has been considered in [6]. A related trend of research dealt with the Casimir force for two Chern insulators, including the full frequency dependence of the conductivity tensor [7]. Interesting results have been obtained also in the context of lattice field theory [8-10], using numerical approaches which are naturally formulated within that context. A noteworthy consequence of having a parity-breaking term manifests itself even for a single mirror. Indeed, this

\footnotetext{
1 'Parity' is understood here in the $2+1$ dimensional sense, namely, the reflection along an odd number of spacetime coordinates.
} 
is the case of the interesting 'quantum Faraday effect' discussed in [11] for the boundary term due to a massive Dirac fermion in $2+1$ dimensions.

In spite of the fact, mentioned in [5], that there is no exact equivalence between boundary conditions and a boundary action, here we show how the results presented in [5] can be obtained by using a judiciously chosen boundary action. As we shall see, it must contain both parity-breaking and parityconserving terms. Interestingly, that is exactly the structure of the leading terms in a small-mass expansion for the effective action due to a massive Dirac field in $2+1$ dimensions [12].

This paper is organized as follows: in Sect. 2 we define the system and present the conventions we have adopted to describe it. Its corresponding Casimir interaction energy is introduced in Sect. 3. In Sect. 4 we consider different particular cases. The reflection coefficients for either one or two mirrors in the basis of right and left circularly polarized states is presented in an appendix. In Sect. 5 we present our conclusions.

\section{The system}

Within the functional integral formalism, which we shall adopt here, it is convenient to define the system in terms of its Euclidean action $\mathcal{S}(A)$, for the Abelian gauge field $A_{\mu}$. We assume $\mathcal{S}(A)$ to have the following structure:

$\mathcal{S}(A)=\mathcal{S}_{0}(A)+\mathcal{S}_{I}(A)$,

where $\mathcal{S}_{0}(A)$ denotes the free EM action:

$\mathcal{S}_{0}(A)=\frac{1}{4} F_{\mu \nu} F_{\mu \nu}, F_{\mu \nu}=\partial_{\mu} A_{\nu}-\partial_{\nu} A_{\mu}, \mu=0,1,2,3$,

and $\mathcal{S}_{I}$ represents the coupling between the field and the mirrors.

We assume, for the time being, that there are just two flat infinite mirrors, located at $x_{3}=0$ and $x_{3}=a$, and denoted by $L$ and $R$, respectively. Since the spatial region occupied by each mirror is a plane, one may interpret $\mathcal{S}_{I}$ as defining two $2+1$-dimensional field theories, involving the components of the gauge field projected to the corresponding reduced spacetime. We recall that, in the case of perfectly conducting mirrors, the role of those $2+1$ dimensional theories is tantamount to imposing the vanishing of the components of the electric field which are parallel to the mirrors, as well as the component of the magnetic field which is normal to them. This can be achieved, for example, by introducing appropriate auxiliary fields which implement those conditions, or by taking the proper limit from certain actions corresponding to imperfect mirrors [13].
In this article, we shall consider a rather general case, obtained by assuming that the corresponding localized actions are quadratic and gauge invariant, but we allow for the existence of both parity-conserving and parity-breaking terms. More explicitly, the form of $\mathcal{S}_{I}$ is

$\mathcal{S}_{I}=\mathcal{S}^{(L)}+\mathcal{S}^{(R)}$

where $\mathcal{S}^{(L, R)}$ denotes the action concentrated on the mirror at $x_{3}=0, a$, respectively. Each one of these terms may contain both parity-even $(e)$ and parity-odd $(o)$ terms. It is convenient to introduce a special notation for the parallel coordinates (including the time $\left.x_{0}\right): x_{\|}=\left(x_{\alpha}\right)$, where indices from the beginning of the Greek alphabet will be assumed to run over the values $0,1,2$. Then we may write formally $\mathcal{S}^{(L)}$, say, as follows:

$\begin{aligned} \mathcal{S}^{(L)} & =\mathcal{S}_{e}^{(L)}+\mathcal{S}_{o}^{(L)}, \\ \mathcal{S}_{e}^{(L)} & =\int \mathrm{d}^{4} x \delta\left(x_{3}\right) \frac{1}{4} F_{\alpha \beta} f_{e}^{(L)}\left(-\partial_{\|}^{2}\right) F_{\alpha \beta} \\ \mathcal{S}_{o}^{(L)} & =\int \mathrm{d}^{4} x \delta\left(x_{3}\right) \frac{i}{2} \varepsilon_{\alpha \beta \gamma} A_{\alpha} f_{o}^{(L)}\left(-\partial_{\|}^{2}\right) \partial_{\beta} A_{\gamma},\end{aligned}$

where $\varepsilon_{\alpha \beta \gamma}$ denotes the Levi-Civita symbol in $2+1$ dimensions, and $f_{e, o}^{(L)}$ have been written as functions of $-\partial_{\|}^{2}$ in order to indicate that they will be, in general, nonlocal kernels in coordinate space. For the $R$ mirror, the structure is quite similar; the relevant changes are that the $\delta$-function must by shifted: $\delta\left(x_{3}\right) \rightarrow \delta\left(x_{3}-a\right)$ and, since the mirrors will not be regarded as necessarily identical in their properties, the kernels may be different. Thus, in $\mathcal{S}^{(R)}$ one also has to make the replacement: $f_{e, o}^{(L)} \rightarrow f_{e, o}^{(R)}$. Note that the kernels will have the mass dimensions: $\left[f_{e}^{(L, R)}\right]=-1$ and $\left[f_{o}^{(L, R)}\right]=0$.

Assuming, however, that the mirrors' properties are translation invariant and time independent (i.e., invariant under translations in the $x_{\|}$coordinates), they will be local in momentum space. Note that the cases of perfect mirrors, or mirrors described purely by a $\mathrm{C}-\mathrm{S}$ term, may be obtained by taking particular limits for the kernels.

We see that, introducing Fourier transformations with respect to the parallel coordinates,

$\widetilde{A}_{\alpha}\left(k_{\|}, x_{3}\right)=\int \mathrm{d}^{3} x_{\|} e^{-i k_{\|} \cdot x_{\|}} A_{\alpha}\left(x_{\|}, x_{3}\right)$.

We may write

$$
\begin{aligned}
& \mathcal{S}_{e}^{(L)}=\frac{1}{2} \int \frac{\mathrm{d}^{3} k_{\|}}{(2 \pi)^{3}} \widetilde{A}_{\alpha}^{*}\left(k_{\|}, 0\right) \alpha_{P}^{(L)}\left(k_{\|}\right) P_{\alpha \beta}\left(k_{\|}\right) \widetilde{A}_{\beta}\left(k_{\|}, 0\right) \\
& \mathcal{S}_{o}^{(L)}=\frac{1}{2} \int \frac{\mathrm{d}^{3} k_{\|}}{(2 \pi)^{3}} \widetilde{A}_{\alpha}^{*}\left(k_{\|}, 0\right) \alpha_{Q}^{(L)}\left(k_{\|}\right) Q_{\alpha \beta}\left(k_{\|}\right) \widetilde{A}_{\beta}\left(k_{\|}, 0\right)
\end{aligned}
$$


where

$\alpha_{P}^{(L)}\left(k_{\|}\right) \equiv k_{\|}^{2} f_{e}^{(L)}\left(k_{\|}^{2}\right), \quad \alpha_{Q}^{(L)}\left(k_{\|}\right) \equiv-\left|k_{\|}\right| f_{o}^{(L)}\left(k_{\|}^{2}\right)$,

and we have introduced

$P_{\alpha \beta}\left(k_{\|}\right)=\delta_{\alpha \beta}-\frac{k_{\alpha} k_{\beta}}{k_{\|}^{2}}, \quad Q_{\alpha \beta}\left(k_{\|}\right)=\varepsilon_{\alpha \gamma \beta} \frac{k_{\gamma}}{\left|k_{\|}\right|}$.

These tensors satisfy algebraic relations which, using a matrix notation, adopt the form

$P^{2}=P, \quad Q^{2}=-P, \quad P Q=Q P=Q$.

To simplify our next developments, it is convenient to have a complete set of orthogonal projectors for the space of $3 \times 3$ Hermitian matrices, which naturally arise in the Fourier representation. The orthogonality property allows one to deal with each invariant subspace separately, naturally decomposing the original problem a set of one-dimensional decoupled problems.

Those projectors can be built by inspection, taking into account the relations above. Indeed, defining $P^{ \pm} \equiv \frac{P \pm i Q}{2}$ and $P^{\prime} \equiv I-P(I$ denotes the identity matrix $)$, we see that

$$
\begin{aligned}
& P^{+}+P^{-}+P^{\prime}=I, \quad\left(P^{ \pm}\right)^{2}=P^{ \pm}, \quad P^{\prime 2}=P^{\prime}, \\
& P^{+} P^{-}=P^{-} P^{+}=P^{ \pm} P^{\prime}=P^{\prime} P^{ \pm}=0 .
\end{aligned}
$$

Then, using the Fourier representation above, we have for the full action $\mathcal{S}$ (in the Feynman gauge) the following expression:

$$
\begin{aligned}
\mathcal{S}(A)= & \frac{1}{2} \int \frac{\mathrm{d}^{3} k_{\|}}{(2 \pi)^{3}} \int \mathrm{d} x_{3}\left\{\tilde{A}_{3}^{*}\left(k_{\|}, x_{3}\right)\right. \\
& \times\left(-\partial_{3}^{2}+k_{\|}^{2}\right) \tilde{A}_{3}\left(k_{\|}, x_{3}\right) \\
& +\tilde{A}_{\alpha}^{*}\left(k_{\|}, x_{3}\right)\left(-\partial_{3}^{2}+k_{\|}^{2}\right) \tilde{A}_{\alpha}\left(k_{\|}, x_{3}\right) \\
& +\tilde{A}_{\alpha}^{*}\left(k_{\|}, x_{3}\right) \delta\left(x_{3}\right)\left[\alpha_{-}^{(L)}\left(k_{\|}\right) P_{\alpha \beta}^{+}\left(k_{\|}\right)\right. \\
& \left.+\alpha_{+}^{(L)}\left(k_{\|}\right) P_{\alpha \beta}^{-}\left(k_{\|}\right)\right] \tilde{A}_{\beta}\left(k_{\|}, x_{3}\right) \\
& +\tilde{A}_{\alpha}^{*}\left(k_{\|}, x_{3}\right) \delta\left(x_{3}-a\right)\left[\alpha_{-}^{(R)}\left(k_{\|}\right) P_{\alpha \beta}^{+}\left(k_{\|}\right)\right. \\
& \left.\left.+\alpha_{+}^{(R)}\left(k_{\|}\right) P_{\alpha \beta}^{-}\left(k_{\|}\right)\right] \tilde{A}_{\beta}\left(k_{\|}, x_{3}\right)\right\},
\end{aligned}
$$

with

$\alpha_{ \pm}^{(L, R)}=\alpha_{P}^{(L, R)} \pm i \alpha_{Q}^{(L, R)}$.

\section{The interaction energy}

The vacuum energy $E$ of the EM field, in the presence of the mirrors, may be written in terms of the Euclidean vacuum transition amplitude $\mathcal{Z}$ for a time evolution of length $T$ :
$E=-\lim _{T \rightarrow \infty}\left(\frac{1}{T} \log \mathcal{Z}\right)$

where $\mathcal{Z}$ can be represented as the functional integral:

$\mathcal{Z}=\int \mathcal{D} A e^{-\mathcal{S}(A)}$.

Translation invariance along the parallel coordinates suggests to use the Fourier transformation implemented in (11) in order to evaluate the functional integral. Besides, the Fourier transformed gauge field may be decomposed, for each set of values of $x_{3}$ and $k_{\|}$in terms of four orthonormal unit vectors, which we will denote by $\hat{e}^{(+)}, \hat{e}^{(-)}, \hat{e}^{(k)}$, and $\hat{e}^{(3)}$. The $\hat{e}^{(3)}$ vector is parallel to the $x_{3}$ axis, i.e., its $\mu$ component is $\hat{e}_{\mu}^{(3)}=\delta_{\mu}^{3}$. The other three vectors are in the orthogonal subspace to the one generated by $\hat{e}^{(3)}$; one of them, $\hat{e}^{(k)}$, points along $k_{\|}$, while $\hat{e}^{( \pm)} \equiv \frac{\hat{e}^{(1)} \pm i \hat{e}^{(2)}}{\sqrt{2}}$, with $\hat{e}^{(1)}$ and $\hat{e}^{(2)}$, orthogonal to $k_{\|}$, are such that $\hat{e}^{(1)}, \hat{e}^{(2)}$ and $\hat{e}^{(k)}$ form a right-handed orthogonal triplet.

Thus, we may decompose $\tilde{A}_{\mu}\left(k_{\|}, x_{3}\right)$ as follows:

$$
\begin{aligned}
\tilde{A}_{\mu}\left(k_{\|}, x_{3}\right)= & C_{+}\left(k_{\|}, x_{3}\right) \hat{e}_{\mu}^{(+)}+C_{-}\left(k_{\|}, x_{3}\right) \hat{e}_{\mu}^{(-)} \\
& +C_{k}\left(k_{\|}, x_{3}\right) \hat{e}_{\mu}^{(k)}+C_{3}\left(x_{\|}, x_{3}\right) \hat{e}_{\mu}^{(3)} .
\end{aligned}
$$

The Fourier transformed action then becomes (we omit the arguments in the coefficients $C$, for the sake of clarity)

$$
\begin{aligned}
\mathcal{S}(A)= & \frac{1}{2} \int \frac{\mathrm{d}^{3} k_{\|}}{(2 \pi)^{3}} \int \mathrm{d} x_{3}\left\{C _ { + } ^ { * } \left[-\partial_{3}^{2}+k_{\|}^{2}\right.\right. \\
& \left.+\delta\left(x_{3}\right) \alpha_{-}^{(L)}\left(k_{\|}\right)+\delta\left(x_{3}-a\right) \alpha_{-}^{(R)}\left(k_{\|}\right)\right] C_{+} \\
& +C_{-}^{*}\left[-\partial_{3}^{2}+k_{\|}^{2}+\delta\left(x_{3}\right) \alpha_{+}^{(L)}\left(k_{\|}\right)\right. \\
& \left.+\delta\left(x_{3}-a\right) \alpha_{+}^{(R)}\left(k_{\|}\right)\right] C_{-} \\
& \left.+C_{k}^{*}\left(-\partial_{3}^{2}+k_{\|}^{2}\right) C_{k}+C_{3}^{*}\left(-\partial_{3}^{2}+k_{\|}^{2}\right) C_{3}\right\} .
\end{aligned}
$$

The action thus becomes the sum for each $k_{\|}$, of four independent actions, each one corresponding to a single degree of freedom, represented by the corresponding coefficient $C$. The functional integration measure factorizes with respect to $k_{\|}$(each value can be treated separately) and also, for each $k_{\|}$, into the product of the measures for each coefficient.

Since $C_{k}$ and $C_{3}$ do not see the mirrors, they can be discarded when evaluating the effect of the mirrors on the vacuum energy. Taking also into account that $\log$ of $\mathcal{Z}$ becomes extensive in $T$ and in the area $L^{2}$ of the mirrors, the energy per unit area, $\mathcal{E}$, becomes

$\mathcal{E}=-\int \frac{\mathrm{d}^{3} k_{\|}}{(2 \pi)^{3}} \log \left[\mathcal{Z}_{k_{\|}}\right]$ 
where

$\mathcal{Z}_{k_{\|}}=\mathcal{Z}_{k_{\|}}^{(+)} \mathcal{Z}_{k_{\|}}^{(-)}$

where each factor above corresponds to the functional integral over the respective coefficient, and may therefore be expressed formally as a functional determinant:

$$
\begin{aligned}
\mathcal{Z}_{k_{\|}}^{( \pm)}= & \left(\operatorname { d e t } \left[-\partial_{3}^{2}+k_{\|}^{2}+\delta\left(x_{3}\right) \alpha_{\mp}^{(L)}\left(k_{\|}\right)\right.\right. \\
& \left.\left.+\delta\left(x_{3}-a\right) \alpha_{\mp}^{(R)}\left(k_{\|}\right)\right]\right)^{-\frac{1}{2}}
\end{aligned}
$$

Finally, taking into account the known results about the functional determinants of the kind arising in the equation above [14], we see that the energy per unit area may be written as follows:

$$
\begin{aligned}
\mathcal{E}=\frac{1}{2} & \int \frac{\mathrm{d}^{3} k_{\|}}{(2 \pi)^{3}} \log \left[\left(1-r_{-}^{(L)} r_{-}^{(R)} e^{-2\left|k_{\|}\right| a}\right)\right. \\
& \left.\times\left(1-r_{+}^{(L)} r_{+}^{(R)} e^{-2\left|k_{\|}\right| a}\right)\right] .
\end{aligned}
$$

We have introduced

$$
r_{ \pm}^{(L, R)}=\frac{\alpha_{ \pm}^{(L, R)}}{\alpha_{ \pm}^{(L, R)}+2\left|k_{\|}\right|}
$$

which play the role of Euclidean reflection coefficients.

It is interesting to note that the energy of the system may be thought of as decoupled between two contributions, each one corresponding to either left or right circular polarization modes. Based on these modes, a useful parametrization of the reflection coefficients (inspired by [5]) is the following:

$r_{+}^{(L)}=-\left|r^{(L)}\right| e^{2 i \delta_{L}}, r_{+}^{(R)}=-\left|r^{(R)}\right| e^{-2 i \delta_{R}}$

(the minus signs amount to a phase convention for $\delta_{L, R}$ ). This allows us to write for the energy

$$
\begin{aligned}
\mathcal{E}= & \frac{1}{2} \int \frac{\mathrm{d}^{3} k_{\|}}{(2 \pi)^{3}} \log \left(1-2\left|r^{(L)}\right|\left|r^{(R)}\right| \cos (2 \delta) \mathrm{e}^{-2\left|\mathrm{k}_{\|}\right| \mathrm{a}}\right. \\
& \left.+\left|r^{(L)}\right|^{2}\left|r^{(R)}\right|^{2} e^{-4\left|k_{\|}\right| a}\right),
\end{aligned}
$$

where $\delta=\delta_{L}-\delta_{R}$.

\section{Results and discussion}

Let us first show how one can recover the result of imposing $\mathrm{C}-\mathrm{S}$ boundary conditions, considered in [5]. That situation involves both parity-breaking and parity-conserving boundary terms, since the boundary conditions mix the parallel components of the electric field with the parallel components of the magnetic field, and the normal component of the magnetic field with the normal component of the electric field.
By inspection of the boundary conditions due to the boundary action we consider in this article, recalling (7), (12), we choose

$f_{e}^{(L)}=f_{e}^{(R)}=-\frac{1}{\left|k_{\|}\right|}$,

$f_{o}^{(L)}=\theta(0), f_{o}^{(R)}=-\theta(a)$,

where the minus sign in the last equation is just to be consistent with the choice made in [5] to introduce the boundary conditions (namely, the normals corresponding to the two surfaces are opposite). Thus,

$r_{L}=-\frac{1+i \theta(0)}{1-i \theta(0)}, \quad r_{R}=-\frac{1-i \theta(a)}{1+i \theta(a)}$

Both have modulus equal to 1 and are therefore pure phases. Defining

$r_{L}=-e^{2 i \delta_{0}}, \quad r_{R}=-e^{-2 i \delta_{a}}$

with $\delta_{0, a} \equiv \arctan \theta(0, a)$, we see that Eq. (20) becomes

$$
\begin{aligned}
\mathcal{E} & =\frac{\varphi_{b}(\delta)}{a^{3}} \\
\varphi_{b}(\delta) & =\frac{1}{32 \pi^{2}} \int_{0}^{\infty} \mathrm{d} k k^{2} \log \left(1-2 \cos (2 \delta) \mathrm{e}^{-\mathrm{k}}+\mathrm{e}^{-2 \mathrm{k}}\right),
\end{aligned}
$$

with $\delta=\delta_{0}-\delta_{a}=\arctan \left(\frac{\theta(0)-\theta(a)}{1+\theta(0) \theta(a)}\right)$, which agrees with the result in [5].

It is worth noting that this choice of boundary term can also be understood as the most general one such that there are no dimensionful constants in its kernels. Indeed, coming back to the form of the boundary action, we see that it can be written (for the $L$ mirror, say) as follows:

$$
\begin{aligned}
S^{(L)}= & \int \mathrm{d}^{4} x \delta\left(x_{3}\right)\left(\frac{1}{4} F_{\alpha \beta}\left(-\partial_{\|}^{2}\right)^{-1 / 2} F_{\alpha \beta}\right. \\
& \left.+\frac{i \theta_{L}}{2} \varepsilon_{\alpha \beta \gamma} A_{\alpha} \partial_{\beta} A_{\gamma}\right) .
\end{aligned}
$$

It is worth noting that essentially the same structure arises as the one-loop effective action for a massless Dirac fermion, with the parity-odd term reflecting the existence of the parity anomaly.

We can also consider boundary terms which only contain parity-breaking terms such that the violation of parity is maximal. This case amounts to taking $f_{e}^{(L, R)}=0$, and $f_{o}^{(L, R)}=\theta_{L, R}$ where each $\theta_{L, R}$ is a dimensionless constant.

The result for $\mathcal{E}$ in this case may be put as follows:

$\mathcal{E}=\frac{\varphi\left(\theta_{L}, \theta_{R}\right)}{a^{3}}$ 
with the dimensionless function $\varphi$ :

$$
\begin{aligned}
\varphi\left(\theta_{L}, \theta_{R}\right)= & \frac{1}{32 \pi^{2}} \int_{0}^{\infty} \mathrm{d} k k^{2} \\
& \times \log \left[1+\frac{\theta_{L}^{2} \theta_{R}^{2}}{\left(4+\theta_{L}^{2}\right)\left(4+\theta_{R}^{2}\right)} e^{-2 k}\right. \\
& \left.+\frac{8 \theta_{L} \theta_{R}-2 \theta_{L}^{2} \theta_{R}^{2}}{\left(4+\theta_{L}^{2}\right)\left(4+\theta_{R}^{2}\right)} e^{-k}\right] .
\end{aligned}
$$

In particular, for identical mirrors $\theta_{L}=\theta_{R} \equiv \theta$,

$$
\begin{aligned}
& \varphi(\theta, \theta) \equiv \varphi_{g}(\theta)=\frac{1}{32 \pi^{2}} \int_{0}^{\infty} d k k^{2} \\
& \quad \times \log \left[1-\frac{\theta^{2}}{\left(4+\theta^{2}\right)^{2}} e^{-k}\left[\theta^{2}\left(2-e^{-k}\right)-8\right]\right] .
\end{aligned}
$$

On the other hand, if the $\mathrm{C}-\mathrm{S}$ coefficients have equal modulus and opposite signs, $\theta_{L}=-\theta_{R} \equiv \theta$,

$$
\begin{aligned}
& \varphi(\theta,-\theta) \equiv \varphi_{u}(\theta)=\frac{1}{32 \pi^{2}} \int_{0}^{\infty} \mathrm{d} k k^{2} \\
& \quad \times \log \left[1-\frac{\theta^{2}}{\left(4+\theta^{2}\right)^{2}} e^{-k}\left[\theta^{2}\left(2-e^{-k}\right)+8\right]\right] .
\end{aligned}
$$

Finally, the result corresponding to a perfect mirror (L) facing a $\mathrm{C}-\mathrm{S}$ mirror $(\mathrm{R})$ with constant $\theta$ may be obtained by evaluating the general expression for the interaction energy for the case $f_{o}^{(L)} \equiv 0, f_{e}^{(L)} \rightarrow \infty$, and $f_{e}^{(R)} \equiv 0, f_{o}^{(L)} \equiv \theta$.
The resulting expression may be put in the form $\mathcal{E}=$ $\frac{1}{a^{3}} \varphi_{c}(\theta)$, with

$$
\varphi_{c}(\theta)=\frac{1}{32 \pi^{2}} \int_{0}^{\infty} \mathrm{d} k k^{2} \log \left[1-\frac{\theta^{2}}{4+\theta^{2}} e^{-k}\left(2-e^{-k}\right)\right]
$$

In order to have a qualitative idea of the behavior of the energy for the different cases we have considered before, we first note that all of them have the same dependence with the distance (since there is no dimensional constant in the problem). They have therefore the structure $\mathcal{E}=\frac{\varphi(\theta)}{a^{2}}$, with a $\varphi$ which may be $\varphi_{g}, \varphi_{u}$ or $\varphi_{c}$, depending on the case considered. The same happens for two perfect conductors, for which we recover the well-known result, given by $\mathcal{E}=\frac{\varphi_{p}}{a^{3}}$, with $\varphi_{p}=-\frac{\pi^{2}}{720}$. Using this constant as a reference, in Fig. 1 we plot a normalized version of $\varphi$ for each case, namely, $\varphi_{n} \equiv \frac{\varphi}{\frac{\pi^{2}}{720}}$, as a function of the $\mathrm{C}-\mathrm{S}$ coefficient $\theta$ for the particular cases considered before. The dotted horizontal line represents the case of two perfect conductors as a reference, the dashed line corresponds to two identical $\mathrm{C}-\mathrm{S}$ mirrors, the solid thin line corresponds to two $\mathrm{C}-\mathrm{S}$ mirrors with opposite sign and equal modulus coefficients, and the solid thick line represents a perfect conductor facing a $\mathrm{C}-\mathrm{S}$ mirror.

Note that, in all $\mathrm{C}-\mathrm{S}$ cases, the energy tends to the one of two perfect conductors as $\theta$ tends to infinity, and their values have no significant difference already after $\theta=50$.

Most notably, for the case of two purely $\mathrm{C}-\mathrm{S}$ mirrors with equal coefficients, we see that the constant becomes negative

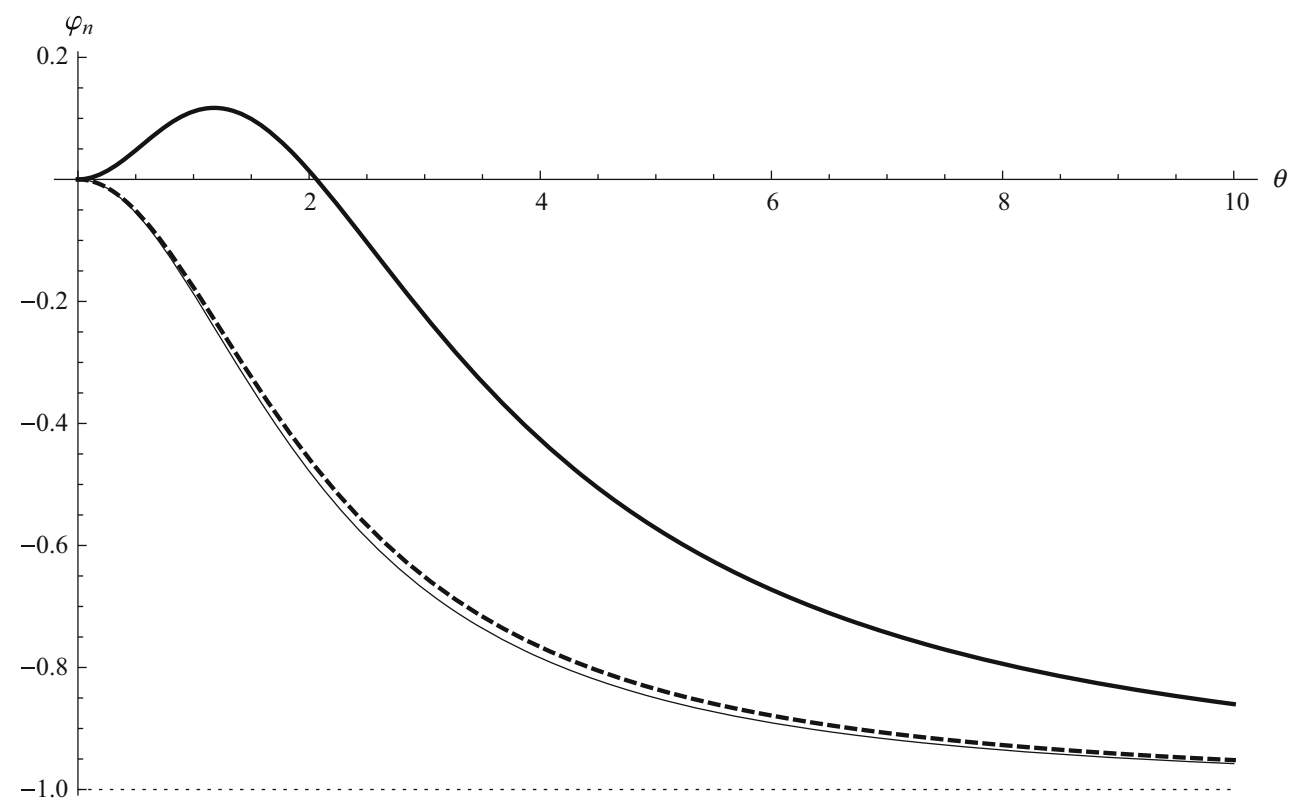

Fig. $1 \varphi_{n}$ vs. $\theta$ for: a two identical C-S mirrors (dashed line), b two C-S mirrors with opposite coefficients (thin line), and $\mathbf{c}$ a perfect conductor facing a $\mathrm{C}-\mathrm{S}$ mirror (thick line). $\varphi_{n}$ for two perfect conductors equals -1 . This is plotted here (as a reference), as the horizontal dotted line at the bottom 
when $\theta$ is lower than $\sim 2.07$, which corresponds to a repulsive force between the mirrors. It exhibits a non-monotonous behavior as a function of $\theta$, which may be understood as a consequence of the fact that, for $\theta$ tending to zero, one should expect the energy to vanish. The existence of another zero at approximately $\theta=2.07$ implies the non-monotonous character of the coefficient.

\section{Conclusions}

We have obtained a general expression for the Casimir energy corresponding to two mirrors, describing matter which may contain both parity-conserving and parity-breaking terms. Based on the general result, we have shown that the results obtained by introducing a boundary condition (rather than boundary action) may be recovered as a result of using a special class of boundary terms. These should involve no dimensionful constant in their definition. As such, they have a very similar structure to the one that one would obtain as the effective action due to a massless Dirac field in $2+1$ dimensions [15].

The general expression that one has for the energy per unit area in the general case, where there may exist both parityconserving and parity-breaking terms (23), shows that only in the case $\delta=0$ the energy becomes the sum of two equal contributions. In other words, the contribution to the vacuum energy of each (left-handed and right-hand) circular polarization mode is the same only when the phases of the reflection coefficients are equal. We have also checked, at the level of the reflection coefficients, that when the relative phase $\delta$ vanishes, the reflection coefficients (see the appendix) become equal for both handednesses, both for one or two mirrors.

In the particular examples we have considered, we have found and interpreted an interesting phenomenon when parity violation is maximal (i.e., no parity-conserving term), namely, the existence of a non-monotonous behavior of the energy as a function of the strength of the $\mathrm{C}-\mathrm{S}$ terms, when assumed to be equal.

Acknowledgements This work was supported by ANPCyT, CONICET, UBA and UNCuyo.

Open Access This article is distributed under the terms of the Creative Commons Attribution 4.0 International License (http://creativecomm ons.org/licenses/by/4.0/), which permits unrestricted use, distribution, and reproduction in any medium, provided you give appropriate credit to the original author(s) and the source, provide a link to the Creative Commons license, and indicate if changes were made.

Funded by SCOAP ${ }^{3}$.

\section{Appendix: Reflection coefficients}

In order to relate the functions and parameters used in the class of models considered to more directly observable mag- nitudes, we calculate here the reflection coefficients for either one or two mirrors.

Reflection coefficients are relevant to a scattering situation, therefore it is rather natural to use here the real-time formalism. Thus we assume in the following the continuation back to real time of the corresponding Euclidean objects has been implemented.

\subsection{One mirror}

Let us first consider the case of only one mirror, located at $x^{3}=0$. The classical equation of motion in this case, assuming the Feynman gauge is used, is given by

$$
A^{\mu}(x)+\delta\left(x^{3}\right) g^{\mu \alpha} \Pi_{\alpha \beta}^{(L)} g^{\beta \mu} A_{\nu}(x)=0,
$$

with

$\Pi_{\alpha \beta}^{(L)} \equiv f_{e}^{(L)}\left(\partial_{\|}^{2}\right)\left(\partial_{\|}^{2} g_{\alpha \beta}-\partial_{\alpha} \partial_{\beta}\right)+f_{o}^{(L)}\left(\partial_{\|}^{2}\right) \epsilon_{\alpha \gamma \beta} \partial^{\gamma}$

(where $g_{\mu \nu}=g^{\mu \nu}=\operatorname{diag}(1,-1,-1,-1)$ and $\left.\partial_{\|}^{2} \equiv \partial_{\alpha} \partial^{\alpha}\right)$.

One can solve the equation above with scattering boundary conditions. We propose a normally incident wave with wave vector $k^{3}=+\sqrt{k_{\|}^{2}} \equiv k$, and we still have the freedom of fixing its polarization (two independent components). It may be seen that the left and right circular polarization vectors diagonalizes the problem in the sense that the corresponding scattering matrices do not mix. Thus, we consider incident waves $\tilde{A}_{I}^{\mu}\left(x^{3}\right)$ such that $\tilde{A}_{I}^{3}=0$, and

$\tilde{A}_{I}^{\alpha}\left(x^{3}\right)=\epsilon_{ \pm}^{\alpha} e^{i k x^{3}}$

We see that, for $x^{3}<0$, the full solution becomes

$\tilde{A}^{\alpha}\left(x^{3}\right)=\epsilon_{ \pm}^{\alpha} e^{i k x^{3}}+r_{ \pm} \epsilon_{ \pm}^{\alpha} e^{-i k x^{3}}$

where the reflection coefficient $r_{ \pm}$, which determines the reflected wave for each polarization, is given by [11]

$r_{ \pm}=\frac{-i \alpha^{\mp}}{2 k+i \alpha^{\mp}}$

where $\alpha^{\mp}$ are the real-time counterparts of the homonymous objects introduced in the Euclidean formalism; namely

$\alpha^{ \pm}=-k^{2} f_{e} \pm f_{o}$.

\subsection{Two mirrors}

The system consists now of the two mirrors. We see that the reflection coefficients for this case are also diagonal in the circular polarization basis. They may be written as follows: 
$r_{ \pm}=\frac{1}{2 i k} \frac{\alpha_{(L)}^{\mp}+\alpha_{(R)}^{\mp} e^{2 i k a}+\frac{i}{2 k} \alpha_{(L)}^{\mp} \alpha_{(R)}^{\mp}\left(1-e^{2 i k a}\right)}{\frac{i}{2 k}\left(\alpha_{(L)}^{\mp}+\alpha_{(R)}^{\mp}\right)-\frac{1}{(2 k)^{2}} \alpha_{(L)}^{\mp} \alpha_{(R)}^{\mp}\left(1-e^{2 i k a}\right)}$.

\section{References}

1. P.W. Milonni, The Quantum Vacuum (Academic Press, San Diego, 1994)

2. K.A. Milton, The Casimir Effect: Physical manifestations of zeropoint energy. World scientific, River edge (2001)

3. M. Bordag, G.L. Klimchitskaya, U. Mohideen, V.M. Mostepanenko, Advances in the Casimir Effect (Oxford University Press, Oxford, 2009)

4. S.M. Girvin, The quantum Hall effect: novel excitations and broken symmetries. Aspects topologiques de la physique en basse dimension. Topological aspects of low dimensional systems, pp. 53-175. Springer, Berlin (1999)

5. M. Bordag, D.V. Vassilevich, Phys. Lett. A 268, 75 (2000). doi:10. 1016/S0375-9601(00)00159-6. arXiv:hep-th/9911179

6. F. Canfora, L. Rosa, J. Zanelli, Phys. Rev. D 84, 105008 (2011). doi:10.1103/PhysRevD.84.105008. arXiv:1105.2490 [hep-th]
7. P. Rodriguez-Lopez, A.G. Grushin, Phys. Rev. Lett. 112(5), 056804 (2014). doi:10.1103/PhysRevLett.112.056804. arXiv:1310.2470 [cond-mat.mes-hall]

8. O. Pavlovsky, M. Ulybyshev, Phys. Part. Nucl. Lett. 7, 345 (2010). doi:10.1134/S1547477110050079

9. O.V. Pavlovsky, M.V. Ulybyshev, Theor. Math. Phys. 164, 1051 (2010)

10. O.V. Pavlovsky, M.V. Ulybyshev, Teor. Mat. Fiz. 164, 262 (2010). doi:10.1007/s11232-010-0084-5

11. I.V. Fialkovsky, D.V. Vassilevich, J. Phys. A 42, 442001 (2009). doi:10.1088/1751-8113/42/44/442001. arXiv:0902.2570 [hep-th]

12. S. Deser, R. Jackiw, S. Templeton, Phys. Rev. Lett. 48, 975 (1982). doi:10.1103/PhysRevLett.48.975

13. C.D. Fosco, F.C. Lombardo, F.D. Mazzitelli, Phys. Rev. D 85, 125037 (2012). doi:10.1103/PhysRevD.85.125037. arXiv: 1203.1855 [hep-th]

14. C. Ccapa Ttira, C.D. Fosco, F.D. Mazzitelli, J. Phys. A 44, 465403 (2011). doi:10.1088/1751-8113/44/46/465403. arXiv:1107.2357 [hep-th]

15. D.G. Barci, C.D. Fosco, L.E. Oxman, Phys. Lett. B 375, 267 (1996). doi:10.1016/0370-2693(96)00224-9. arXiv:hep-th/9508075 